\title{
Konversationsanalytische Medienforschung
}

\author{
Ruth Ayaß
}

In den vergangenen Jahren hat eine Fülle konversationsanalytischer Studien die Kommunikation in oder mittels oder über Massenmedien zum Gegenstand gemacht. So liegen, um nur einige Beispiele zu nennen, Untersuchungen über „recipient design“ und „turn"-Strukturen von Nachrichteninterviews vor oder über Beteiligungsstrukturen in so genannten "radio call ins". Der deutschen (und auch internationalen) Medienforschung sind diese Untersuchungen bislang nabezu unbekannt. Der Beitrag stellt die bisherigen Ergebnisse und Gegenstände konversationsanalytischer Untersuchungen zu massenmedialer Kommunikation in Methode und Inhalt vor. Ziel des Beitrags ist, (massen-)mediale Kommunikation als Gegenstand der Konversationsanalyse methodisch und methodologisch zu reflektieren, sie als Methode vorzustellen und damit den Methodenkanon der Medienforschung zu erweitern.

Keywords: Konversationsanalyse, ethnomethodologische Konversationsanalyse, mediale Gattungen, Rezeptionsforschung, qualitative Methoden, Nachrichteninterview, Computer Supported Cooperative Work

\section{Einleitung 1}

Die Medienforschung zeichnet sich erfreulicherweise durch eine nahezu unüberschaubare Pluralität der Methoden aus. In den letzten Jahren sind neben die klassischen quantitativen Verfahren, wie zum Beispiel die Inhaltsanalyse, eine Fülle qualitativer Verfahren getreten. Dennoch schöpft die Medienforschung das vorhandene Methodenrepertoire der qualitativen Methodologie nicht aus. Der vorliegende Beitrag möchte eine qualitative Methode diskutieren, die in den letzten Jahren mehr und mehr Anwendung auf massenmediales Material findet, die ethnomethodologische Konversationsanalyse. Bislang wird sie in der Medienforschung nur wenig wahrgenommen - weder als Methode noch in ihren empirischen Resultaten. So ist sie zum Beispiel im von Jensen und Jankowski herausgegebenen „Handbook of qualitative methodologies for mass communication research" (1991, seither mehrere Reprints) nicht vertreten (und wird auch nicht erwähnt). Und auch im neueren „Handbook“ $(2002,108)$ findet sie mit gerade drei Sätzen eine eher randständige Beachtung. Den empirischen Untersuchungen, die sich mit medialer Kommunikation befassten, erging es nicht viel anders. Die Untersuchungen von John Heritage, Steven Clayman, Paddy Scannell oder David Greatbatch zu Fernsehnachrichten z. B. sind, nicht nur in der allgemeinen (deutschen) Medienforschung, sondern auch und gerade in der Nachrichtenforschung praktisch unbekannt - und dies, obwohl sie nicht gerade in besonders auf Konversationsanalyse spezialisierten Zeitschriften, sondern eher an allgemein prominenten Orten publiziert wurden (z. B. in

1 Für die Lektüre und Kommentierung früher Fassungen dieses Aufsatzes in verschiedenen Stadien seiner Entstehung danke ich Jörg Bergmann, Jessika Braun, Antonia Krummheuer, Veronika Tacke und Karola Pitsch (alle Universität Bielefeld). 
„American Sociological Review“, in „Social Problems“ oder im „Handbook of Discourse Analysis"). ${ }^{2}$

Im Gegenzug wird auch in vielen konversationsanalytischen Untersuchungen die aktuelle mediensoziologische bzw. medienwissenschaftliche Forschung eher als pauschaler Verweis, zum Beispiel in Form einer Sammelfußnote, abgehandelt und im weiteren Text nicht näher thematisiert. Von wenigen Ausnahmen abgesehen, kann man von einer wechselseitigen Ignoranz sprechen. Ethnomethodologie und Konversationsanalyse sind an diesem Zustand, wie noch zu zeigen sein wird, nicht gerade unschuldig. Dabei könnten beide Disziplinen voneinander profitieren, wie im Folgenden anhand der vorliegenden konversationsanalytischen Untersuchungen massenmedialer Kommunikation gezeigt wird. Denn die Konversationsanalyse hat nicht nur eine Fülle empirischer Untersuchungen zu klassischen Massenmedien vorgelegt, sie ist zudem im Moment vor allem in einem neuen Gebiet, an dem sie maßgeblich beteiligt ist, der so genannten „Computer Supported Cooperative Work“ (CSCW), ein ausgesprochen innovativer Ansatz, der sich auch intensiv den neuen Medien widmet.

Der vorliegende Beitrag konzentriert sich im Wesentlichen auf die Ergebnisse und Gegenstände konversationsanalytischer Untersuchungen zu klassischer massenmedialer Kommunikation, doch auch andere Bereiche aus der Ethnomethodologie, wie etwa die CSCW, werden berührt. ${ }^{3}$ Ziel dieses Artikels ist es, die Konversationsanalyse als Methode der deutschen Medienforschung vorzustellen, (massen-) mediale Kommunikation als Gegenstand der Konversationsanalyse methodisch und methodologisch zu reflektieren, und damit den Methodenkanon der Medienforschung zu erweitern.

Abschnitt 2 erläutert die wesentlichen Prämissen der Konversationsanalyse und grenzt sie von der linguistischen Gesprächsanalyse ab. Abschnitt 3 präsentiert eine Übersicht über die wesentlichen Resultate der konversationsanalytischen Produkt-, Produktions- und Rezeptionsstudien. Abschnitt 4 behandelt den Bereich, zu dem mit Abstand am meisten Studien vorliegen: Nachrichteninterviews. Beide Abschnitte skizzieren die Untersuchungen zunächst eher allgemein, konzentrieren sich dann aber, um die konkrete Anwendung zu veranschaulichen, jeweils auf einzelne empirische Analy-

2 Im englischen Sprachraum ist die Situation nur unwesentlich besser. Zwar wurden einige wenige konversationsanalytische Untersuchungen zu Massenmedien in klassischen medienwissenschaftlichen Zeitschriften publiziert, doch beschränkt sich dies im Wesentlichen auf ein 1986 in „Media, Culture \& Society“ publiziertes Heft zu „Broadcast talk“ (hrsg. von Scannell 1986). Vereinzelt erschienen später, wiederum in „Media, Culture \& Society“, weitere Aufsätze, die zur Konversationsanalyse gezählt werden können (Roth 1998, Myers 2000). Auch ein Sonderheft von „Research on Language and Social Interaction“, in dem ein Themenschwerpunkt über ein besonderes Fernsehereignis („The Dan Rather/George Bush Episode on CBS News“; mit Beiträgen von Schegloff, Clayman/Whalen, Nofsinger und Pomerantz; alle 1988/89) erfolgte, wurde in der Medienforschung, auch der englischsprachigen, praktisch ignoriert. Einem von Thornborrow herausgegebenen „Special Issue“ der (auch) konversationsanalytisch ausgerichteten Zeitschrift „Text“ (unter anderem mit Beiträgen von Hutchby über „public debates“, Scannell über Dokumentarfilme und Thornborrow über Talkshows; alle 1997) erging es nicht viel anders.

3 Diese Konzentration auf die Methode der Konversationsanalyse führt auch dazu, dass andere qualitative Methoden mit anderen theoretischen Hintergründen wie etwa die Studien, die mit ethnographischen Methoden arbeiten, oder Untersuchungen, die sich auf den symbolischen Interaktionismus beziehen, wie zum Beispiel die Untersuchungen von James Lull, Norman Denzin, Thomas Lindlof und anderen, hier nicht weiter thematisiert werden. 
sen. Der fünfte und letzte Abschnitt diskutiert Anforderungen an eine konversationsanalytisch fundierte Medienforschung.

\section{2. $\mathrm{Zu}$ Methode und Gegenstandsbereich der Konversationsanalyse}

Die Konversationsanalyse, in ihrer ethnomethodologischen Ausprägung, entstand aus einem eigentümlichen Umstand heraus. Harvey Sacks, der in den sechziger Jahren mit seinen „Lectures“ (posthum 1992) die Konversationsanalyse ins Leben rief, beschrieb ihre Entstehungsgeschichte wie folgt:

"It was not from any large interest in language or from some theoretical formulation of what should be studied that I started with tape-recorded conversations, but simply because I could get my hands on it and could study it again and again, and also, consequentially, because others could look at what I had studied and make of it what they could, if, for example, they wanted to be able to disagree with me." $(1984 a, 26)$

Offensichtlich war es, wissenschaftshistorisch gesehen, ein zufälliges Ereignis, dass dieses Forschungsunterfangen sich direkt der Untersuchung mündlicher Kommunikation widmete, also aus der Ethnomethodologie heraus die Konversationsanalyse als eine Konversationsanalyse entstand. ${ }^{4}$ Harvey Sacks war Schüler von Harold Garfinkel, welcher seinerseits in Fortsetzung der theoretischen (und durchaus einander widersprechenden) Ansätze von Alfred Schütz und Talcott Parsons die Ethnomethodologie als eigenständige soziologische Disziplin entwickelte. ${ }^{5}$ Ethnomethodologie bezeichnet eine Herangehensweise, die nicht nur allgemein den Konstruktionscharakter sozialer Wirklichkeit hervorhebt, sondern das Augenmerk auf deren Herstellung in sozialen Handlungen legt. Soziale Wirklichkeit meint nicht nur die Orientierung von Handelnden an gegebenen Ordnungen, wie etwa Institutionen oder sozialen Dingen. Die soziale Ordnung selbst wird von Handelnden permanent hergestellt, erzeugt und fortgeführt. Soziale Wirklichkeit ist, so Garfinkel, ein „ongoing accomplishment“, eine Vollzugswirklichkeit:

„(..) in contrast to certain versions of Durkheim that teach that the objective reality of social facts or sociology's fundamental principle, the lesson is taken instead and used as study policy, that the objective reality of social facts as an ongoing accomplishment of the concerted activities of daily life, with the ordinary artful ways of that accomplishment being by members known, used, and taken for granted, is for members doing sociology, a fundamental phenomena." (Garfinkel $1967 b$, vii)

Garfinkel selbst hatte in mehreren Untersuchungen diesen Herstellungscharakter sozialer Ordnungen aufgezeigt, etwa in der spektakulären Untersuchung „Passing“ über die Transsexuelle „Agnes“ (1967a). Garfinkel konnte zeigen, dass und wie Agnes, so ihr Pseudonym, ihr Geschlecht fortwährend erzeugt, ja im Handeln erst erwirbt. Die Untersuchung gilt heute als Klassiker der Genderforschung. Ihre Quintessenz - Geschlecht

4 Zur Entstehung der Konversationsanalyse aus der Ethnomethodologie vgl. Bergmann (1988/2), Bergmann (2000c) und Schegloff (1992). Zu einer umfassenden Darstellung zu Methoden und Techniken der Konversationsanalyse siehe ten Have (1999), für eine deutschsprachige Darstellung siehe Deppermann (1999).

5 Zur Entstehungsgeschichte der Ethnomethodologie vgl. auch Bergmann (1988/1), Heritage (1984) sowie Bergmann (2000a; 2000b). 
ist eine interaktive Herstellungsleistung und keine ontologische Gegebenheit - antizipierte die heute in der Genderforschung unumstrittene These des „doing gender" um Jahrzehnte. In anderen Untersuchungen zeigte Garfinkel anhand der so genannten „Krisenexperimente" die Brüchigkeit sozialer Ordnungen auf (1967b, 42-44). Er leitete seine Studenten an, alles als selbstverständlich Hingenommene einer sozialen Situation einzuklammern - und sich zum Beispiel zu Hause wie Fremde zu benehmen. Bekannt geworden sind vor allem die Krisenexperimente, in denen man eine Floskel wie „Wie geht's dir" wörtlich nimmt und beginnt, genaue Nachfragen zu stellen. Die Situationen eskalierten regelmäßig binnen Kürze bis hin zum Kommunikationsabbruch und zeigten die unhinterfragten Voraussetzungen alltäglicher Kommunikation auf, wie das folgende Beispiel für ein solches Krisenexperiment zeigt (1967a, 44):

„The victim waved his hand cheerily.

(S) How are you?

(E) How am I in regard to what? My health, my finances, my school work, my peace of mind, my ...?

(S) (Red in the face and suddenly out of control.) Look! I was just trying to be polite. Frankly, I don't give a damm how you are.“

Für die Ethnomethodologie (wie auch für die aus ihr hervorgehende Konversationsanalyse) ist, um den Herstellungscharakter sozialer Wirklichkeit aufzuzeigen, der Begriff des „doing“ zentral. Sacks hatte zum Beispiel in „On doing , being ordinary““ (1984) gezeigt, wie ,being ordinary“ hergestellt wird. Lindsey Churchill fasst dieses Konzept wie folgt zusammen:

"The ethnomethodologist continually asks the technical question, ,How is that social activity done?" Harvey Sacks keeps this question in front of him by using the verb ,to do' in thinking about social activities. He refers to arguing as doing arguing; being embarrassed as doing embarrassment; exclaiming as doing exclaiming; questioning as doing questioning, etc. In this way he tried to keep focused on the methodological ways in which social activities are produced by members of the culture." $(1971,183)$

Das Material der ersten empirischen Untersuchungen der Konversationsanalyse entstammt vorwiegend aus Alltagsgesprächen, die auf Tonband aufgezeichnet wurden. Als empirischer Forschungsgegenstand war ein solches Material zu diesem Zeitpunkt, zu dem zum Beispiel die Sprechakttheorie mit hypothetischen, in der Regel vom Forscher erfundenen, Sätzen arbeitete, völlig ungewöhnlich. Einige der frühen Untersuchungen stützen sich vorwiegend oder ausschließlich auf Telefongespräche (z. B. Schegloff/Sacks 1973; Schegloff 1972). Der Vorteil dieser speziellen Kommunikationssituation liegt auf der Hand: Sämtliche nonverbale Mitteilungsressourcen, die in Face-to-Face-Begegnungen eine große Rolle für die Kommunikation spielen und die bei Tonbandaufnahmen den Forschern üblicherweise verloren gehen, stehen beim Telefonieren auch den Interagierenden nicht zur Verfügung.

„We have proceeded under the assumption (...) that insofar as the materials we worked with exhibited orderliness, they did not so only for us (...) but for the coparticipants who had produced them. If the materials (records of natural conversations) were orderly, they were so because they had been methodically produced by members of the society for one another (...).“ (Schegloff/Sacks 1973, 290)

Dieser für die Konversationsanalyse schon fast 'chronische' Ordnungsverdacht mündet bei Sacks (1984 a, 22) in die methodische Leitlinie:

"That is, we may alternatively take there is order at all points."

Die Geordnetheit, die Interagierende in ihren Konversationen erzeugen und welche die 
Konversationsanalyse in ihren frühen, mittlerweile als klassisch zu bezeichnenden Analysen zutage brachte, sind weitgehend bekannt: Die Aushandlung von Redeübergaben, die Ordnung von Redezügen in Sequenzen (Sacks/Schegloff/Jefferson 1974), die Präferenzstrukturen von Meinungsäußerungen (Pomerantz 1984), Reparaturen und Korrekturen (Jefferson 1987), die Techniken, sich Aufmerksamkeit zu verschaffen und aufrechtzuerhalten, die interaktive Konstruktion von Äußerungen und der Redezug als interaktive Einheit (Schegloff 1982), Überlappungen, Unterbrechungen, die kollaborative Herstellung von Äußerungen, „duetting“ und „floor fights“, Lachpartikel etc.

Im Zentrum der konversationsanalytischen Untersuchungen stehen „turns“, Redezüge, deren interaktive Hervorbringungen und sequenzielle Ordnungen analysiert werden. Zentral dabei ist der Begriff der Paarsequenz (,adjacency pair“). Paarsequenzen bilden sozusagen die Kleinstform einer sequenziellen Ordnung. Durch die Formulierung des ersten Teils einer Paarsequenz (einer Frage, eines Grußes etc.) werden sequenzielle Erwartungen an die Formulierung des zweiten Teils eröffnet (eine Antwort, ein Gegengruß etc.). Die Konversationsanalyse spricht hier von „konditioneller Relevanz“. Ein weiterer wesentlicher Begriff bildet das „recipient design“. Unter „recipient design“ wird der spezifische Zuschnitt einer Äußerung auf ihren Adressaten verstanden.

In allen Untersuchungen ist der Kerngedanke tragend, dass möglicherweise kein Element, und sei es noch so unscheinbar und klein, zufällig ist. Im Unterschied zu anderen Methoden der Soziologie sowie der Sprachwissenschaft hat die Konversationsanalyse kein ausformuliertes methodisches Regelwerk vorgelegt. Genau genommen lehnt sie eine Kanonisierung ihres methodischen Vorgehens strikt ab. Dennoch lassen sich wesentliche methodische Prämissen benennen: Das Material entstammt authentischen, natürlichen Interaktionssituationen. Unabdingbar ist dabei die Konservierung dieser Interaktionen als Aufzeichnung. Typisch für die Konversationsanalyse ist die Überführung des Materials in - für Neulinge aufgrund ihres Detaillierungsniveaus mitunter schwer lesbare - Transkriptionen, welche auch Lacher, Versprecher, Abbrüche, Dehnungen etc. enthalten. Anhand dieser Transkripte, die die Materialfülle der Aufzeichnung nicht vorab vereinfachen, werden geordnete Strukturen destilliert. Entsprechend den Prämissen eines qualitativen Forschungsprozesses erfolgt diese Interpretation als offenes Verfahren, das nicht hypothesengeleitet vorgeht, sondern Typen und Strukturhypothesen aus dem Material heraus entstehen lässt und im Sinne eines hermeneutischen Zirkels in einem Wechsel von Vorverständnis und Auseinandersetzung mit dem Material zu einer Überwindung jenes Vorverständnisses und damit zu neuen Resultaten kommt.

Die Konversationsanalyse ist personell und institutionell, sei es über Projekte, Publikationen oder gemeinsame Forschungsgegenstände, mit der linguistischen Gesprächsanalyse verwandt. Dennoch existieren klare Unterschiede. ${ }^{6}$ Am deutlichsten lassen sich die Unterschiede an der Relevanz und am Stellenwert des Materials darstellen. Während für die linguistische Gesprächsanalyse das sprachliche Material per Definition der Disziplin primär ist und zentral bleibt, ist für die Konversationsanalyse das sprachliche Material nur eine von vielen Ressourcen, mittels derer die Ordnungsgenerierung durch die Interagierenden eben besonders deutlich aufgezeigt werden kann. Deutlich wird dieser Unterschied gegenwärtig darin, dass die Konversationsanalyse neben ihrem

$6 \mathrm{Zu}$ einer gesprächslinguistischen Kritik der Konversationsanalyse siehe Klemm (2000, 136-144). 
ursprünglichen Gegenstand - gesprochene Sprache alltäglicher Interaktionen - zunehmend auch die visuelle Ebene von Interaktionen und den Umgang mit Artefakten einbezieht. Doch die Differenz soll nicht überzeichnet werden und wird teils von den Betroffenen selbst auch nicht streng wahrgenommen. Schegloff et al. haben jüngst (2002) in der Zeitschrift „Applied Linguistics“ auf die engen Verbindungen zwischen beiden Disziplinen hingewiesen, indem sie die gemeinsamen Forschungsgegenstände hervorhoben, jedoch auch die unterschiedlichen Fragestellungen und Methoden betonten, mit denen Konversationsanalyse einerseits und interaktionale Linguistik andererseits vorgehen. Selting/Couper-Kuhlen (2001) betonen im Gegenzug in ihrem „Forschungsprogramm ,Interaktionale Linguistik““ explizit die enge Verbindung zwischen (sprachwissenschaftlicher) interaktionaler Linguistik und (ethnomethodologischer) Konversationsanalyse: Eine Kooperation sei „unverzichtbar“, die Grenzen „müssen fließend bleiben“, und: „Eine genauere Abgrenzung ist unnötig.“ $(2001,281)^{7}$

Ein weiter gehender Unterschied lässt sich im theoretischen Selbstverständnis ausmachen. Die im engeren Sinn soziologische Konversationsanalyse betont ihren ethnomethodologischen Hintergrund. Dabei kommt es immer wieder zu Auseinandersetzungen mit der Ethnomethodologie, von der sie abstammt. Im Kern geht es darum, dass die Ethnomethodologie den Konversationsanalytikern vorwirft, sie hätten ihr ethnomethodologisches Erbe an die Sequenz verraten und seien Strukturfetischisten geworden. Garfinkel selbst bezeichnete die Konversationsanalyse einmal despektierlich als „latter day CA“ und die Konversationsanalytiker als „structure’s mandarins“, als „Mandarine der Struktur“ (also „Strukturbeamte“). Diese Konflikte flammen immer wieder auf, wie etwa im mehrteiligen, recht polemischen Austausch zwischen Schegloff und Billig in der Zeitschrift „Discourse and Society“ (Schegloff 1999a, Billig 1999a, Schegloff 1999b, Billig 1999b). ${ }^{8}$

Von diesem Disput bleibt die Gesprächsanalyse unbehelligt. Möglicherweise lässt sich die sonst meist eher diffuse Trennlinie zwischen (linguistischer) Gesprächsanalyse und (ethnomethodologischer) Konversationsanalyse anhand dieser Debatte ziehen: Wer

7 Im Folgenden wird in erster Linie auf die im engeren Sinn konversationsanalytischen Arbeiten Bezug genommen. Rekurse auf gesprächsanalytische Arbeiten erfolgen nur sporadisch und auch nur, wenn in den Untersuchungen selbst inhaltlich oder methodisch ein klarer Zusammenhang zur Konversationsanalyse hergestellt wird.

8 Die jüngste Diskussion um die Rolle der Konversationsanalyse wurde - weitgehend einvernehmlich - im Jahr 2000 in der Zeitschrift „Text“ zwischen Michael Lynch (2000a; 2000b) und Wes Sharrock (2000) geführt, eine Erörterung mit - zumindest für Konversationsanalytiker fast skurrilen Zügen, da sie darin gipfelt, das Ende der Konversationsanalyse auf das Erscheinungsjahr des für die Konversationsanalyse grundlegenden (und sie in der Wahrnehmung seitens der Konversationsanalytiker begründenden) Textes „A simplest systematics“ (Sacks/ Schegloff/Jefferson 1974) sowie den frühen Tod von Harvey Sacks 1975 zu datieren - und zugleich nicht einen weiteren konversationsanalytischen Text zu zitieren. Für Außenseiter sind diese Texte hingegen vermutlich völlig unverständlich. Die Folgen dieses Zerwürfnisses sind aber gerade für die Außenwahrnehmung nicht zu unterschätzen. Und auch für die Medienforschung zeitigt dieser Disput gewisse Konsequenzen, da - auf beiden Seiten - ein wechselseitiges Beschweigen von Publikationen erfolgt. So werden in dem von Jalbert herausgegebenen Band „Media Studies. Ethnomethodological approaches“ (1999) zum Beispiel die über Medien arbeitenden Konversationsanalytiker quasi exkommuniziert, wenn sie weder als Autoren auftauchen noch auch nur ansatzweise referiert werden - bis hin zur klammheimlichen Umbenennung der Konversationsanalyse in „sequential analysis“. 
sich davon nicht angesprochen (oder gar angegriffen und falsch dargestellt) fühlt, ist vermutlich eher Gesprächs- denn Konversationsanalytiker.

In den 80er Jahren beginnt sich der Gegenstand der Konversationsanalyse in zwei Punkten entscheidend zu verändern, die, wie zu zeigen sein wird, gravierende Auswirkungen auf Methode und Material der Untersuchungen hatten und haben. Mit den Untersuchungen von Goodwin (1981) und Heath (1986), die erstmals Videoaufzeichnungen statt Tonbandaufnahmen einsetzten, änderte sich zunächst der Materialtyp der Studien, i.e. die Medialität des Materials, radikal. Zum Zweiten änderte sich der Gegenstand der Untersuchungen. Lagen bislang mit Telefongesprächen vielfach Daten aus alltäglichen, geselligen, nicht-institutionellen Situationen vor, galt die Aufmerksamkeit mehr und mehr Kommunikationen aus institutionellen Kontexten - Medizin, Therapie, Gericht, Schule, Massenmedien (für eine umfassendende und aktuelle Literaturübersicht vgl. Schegloff et al. 2002, 10-12). ${ }^{9}$ Der massenmediale Kontext zeichnet sich gegenüber anderen institutionellen Kontexten im Wesentlichen dadurch aus, dass seine Interaktionen an abwesende Zuhörer respektive Zuschauer gerichtet und auf sie zugeschnitten sind (Heritage/Greatbatch 1991). Zu den ersten Studien aus der Konversationsanalyse, die sich explizit mit Material aus massenmedialer Kommunikation befassen, zählen zum Beispiel Max Atkinsons „Our masters’ voices“, eine Untersuchung, welche die rhetorischen Strategien und Techniken der Applaus-Elizitierung in Politikerreden aufzeigt (1984), sowie John Heritages Aufsatz „Analyzing news interviews. Aspects of the production of talk for an overhearing audience“ (1985). ${ }^{10}$

In den letzten Jahren hat die Konversationsanalyse weiteres neues Terrain entdeckt. Sie untersucht mittlerweile auch technisch oder medial (im weitesten Sinne) vermittelte Kommunikationssituationen, etwa in der Medizin, wo an Monitoren oder Geräten Ultraschallbilder, Röntgenaufnahmen oder EKG-Verlaufskurven verhandelt werden, oder im Cockpit, wo die geographischen oder meteorologischen Daten wie Luftdruck oder Bodenabstand nicht über persönliche Anschauung erworben, sondern über technische Anzeigen „gelesen“" werden. Alles in allem hat eine deutliche Medialisierung des Gegenstands einerseits und eine Visualisierung des konversationsanalytischen Materials andererseits stattgefunden.

Die vorliegenden empirischen Untersuchungen lassen sich pragmatisch in drei (einander nicht vollständig exklusive) Gruppen einteilen. Sie unterscheiden sich im Wesentlichen in der dem Forschungsgegenstand inhärenten Medialität einerseits bzw. Grad und Art der Medialität des untersuchten Materials andererseits.

1. auditive oder audiovisuelle Aufzeichnungen von Face-to-Face-Interaktionen in natürlichen oder institutionellen Kontexten. Viele Untersuchungen der Konversationsanalyse zählen nach wie vor zu diesem Typ. Hier finden sich aktuelle Untersuchungen zu klassischen Themen der Konversationsanalyse wie z. B. Heritages neuere Untersu-

9 Wobei dies als Paradigmenwechsel der Konversationsanalyse zu interpretieren, wie es vielfach erfolgt und der Analyse alltäglicher, nicht-institutioneller Kommunikation den Ruf der „classic“ oder „real CA“ einbrachte, historisch nicht völlig korrekt ist: Die ersten Untersuchungen (Sacks 1972 und Schegloff 1972) arbeiteten mit Material aus institutionellen Kontexten, einer Telefonseelsorge („suicide prevention hotline“; Sacks) und Polizeinotrufen (Schegloff).

10 Auch hier existieren mit den frühen Untersuchungen über Schlagzeilen von z. B. Schenkein (1979) und Lee (1984) historische Vorläufer. Da in ihnen der Fokus weniger auf dem massenmedialen Kontext liegt als vielmehr auf den so genannten „membership categories“, werden sie an anderer Stelle kurz behandelt (Ende des Abschnitts 3.3). 
chung zur Partikel „oh“ (1998) oder Schegloffs umfassende Untersuchung über Unterbrechungen und Überlappungen (2000). Aber auch die Konversationsanalyse, die sich auf Face-to-Face-Begegnungen konzentriert, behandelt neue Themenfelder, wie etwa interkulturelle Kommunikationen zwischen „native speaker“ einer Sprache und „nonnative speaker" (vgl. z. B. Günthner 1993). Zunehmend werden hier Videoaufzeichnungen anstatt Tonbandaufzeichnungen eingesetzt (z. B. Egbert 1996).

Für die Medienforschung scheinen diese Untersuchungen zunächst weiter keine große Rolle zu spielen. Dies ist bei den folgenden Themen sicherlich nicht der Fall. Sie berühren, mit ihren Untersuchungen über alte und neue Medien, zentrale Gegenstände der Medienforschung. Ihnen gilt daher im Folgenden die Aufmerksamkeit:

2. medial vermittelte Kommunikationen zwischen zwei oder mehreren Teilnehmern. Hierunter zählen die Untersuchungen der „studies of work“, Computer Mediated Communication (CMC), Human Computer Interaction (HCI) sowie die eingangs erwähnten Studien der Computer Supported Cooperative Work (CSCW), an denen Konversationsanalytiker und Ethnomethodologen entscheidend mitwirken (als Klassiker gilt Lucy Suchmans „Plans and situated actions“, 1987). Des Weiteren fallen hierunter eine Reihe von Untersuchungen zu den so genannten „neuen“ Medien: etwa im Chat (IRC), elektronische Kommunikationen (E-Mail; Mailinglisten; Bulletin Board Systems) etc. (vgl. für deutsche vorwiegend gesprächsanalytische Beiträge z. B. die Untersuchungen in Thimm 2000).

In diesem Gebiet hat im vergangenen Jahrzehnt insbesondere in der CSCW eine geradezu explosionsartige Entwicklung stattgefunden, die vor allem in Deutschland über die Konversationsanalyse hinaus bislang kaum wahrgenommen wird. Die CSCW etwa ist ein innovativer und hochproduktiver Ansatz, der seit Jahren zum Beispiel eine eigene Zeitschrift führt. Als exemplarische Untersuchungen lassen sich die Arbeiten der Goodwins (Goodwin 1996, Goodwin/Goodwin 1996) und von Suchman (1993) nennen, in denen die visuellen und medialen Interpretationsleistungen der Lotsen in einem Airporttower aufgezeigt werden; oder die Studie von Whalen et al. (2002), die die „teleservice work " in einem Call Center untersuchen. Ein weiteres beeindruckendes Beispiel ist die Studie von Heath/Hindmarsh/Luff (1999), in der die „dislocated world“ der mit Anzeigen und elektronischen Geräten arbeitenden Londoner U-Bahn-Zugführer analysiert wird. Exemplarisch sind für den deutschen Sprachraum die konversationsanalytischen Falluntersuchungen von Meier zu Strukturmerkmalen von Videokonferenzen (2000a; 2000b; 2002; 2003) sowie Goll (2002) über ein virtuell arbeitendes Unternehmen, das vorzugsweise über ein firmeneigenes Gruppenprogramm und Intranet kommuniziert. Meier zeigt, wie sich bei Videokonferenzen die Verteilung auf verschiedene Standorte auf die Interaktion auswirkt, wie z. B. die „Trägheit“ der Tonübertragungen zu problematischen Turnübergaben mit Überlappungen, gleichzeitigen Abbrüchen und „re-starts" führt. ${ }^{11}$ Goll (2002) beleuchtet das Zusammenspiel der verschiedenen Medien in einem räumlich verteilten Unternehmen. Sie zeigt, wie die teils weit voneinander entfernt arbeitenden Firmenangehörigen eine „virtuelle Präsenz“ herstellen - und welche enorme Rolle ausgerechnet informelle Kommunikation für die Mitarbeiter spielt. Die Grenzen der CSCW zu den so genannten „workplace studies“ sind fließend (vgl. Button 1993, Luff/Hindmarsh/Heath 2000, zu einer kurzen deutschen Darstellung siehe Bergmann 2000a).

11 Heath und Luff sprachen schon 1993 bezüglich der medialen Ko-Präsenz bei Videokonferenzen von einer verflachten, entkörperten („disembodied“) Wahrnehmung. 
3. massenmediale Kommunikationen (im eigentlichen Sinn des Wortes, wie er bislang in der Medienforschung gebraucht wird): die „one-to-many“-Kommunikationen mittels der Massenmedien Hörfunk, Zeitung, Fernsehen, Bildschirmtexte, Film, Plakate, Buch, Flugblatt etc. Die Grenzen zur zweiten Gruppe sind, insbesondere, was mit Inter- und Intranet und Bulletin Boards und E-Mail-Verteilern klassische „one-to-many“Kommunikationssituationen betrifft, fließend. Ethnomethodologie und Konversationsanalyse haben eine Fülle von empirischen Untersuchungen hierzu hervorgebracht. Auf sie konzentrieren sich die beiden folgenden Abschnitte.

\section{Konversationsanalytische Untersuchungen massenmedialer Kommunikation}

Konversationsanalytische Untersuchungen von massenmedialer Kommunikation sind, von wenigen noch zu erwähnenden Ausnahmen abgesehen, Analysen von medialen Produkten, darunter insbesondere Nachrichtenkommunikation. Bevor die wesentlichen Resultate dieser Untersuchungen dargestellt werden, seien zunächst jene Untersuchungen genannt, die sich mit der Produktion und Rezeption massenmedialer Kommunikation auseinander setzen.

\subsection{Produktionsanalysen}

Zur Produktion massenmedialer Erzeugnisse liegen nur wenige Analysen vor. Während Meier (1997) Arbeitsbesprechungen im Allgemeinen untersucht (zu seinem Datenkorpus zählen jedoch auch Videoaufzeichnungen von Redaktionskonferenzen eines Radiosenders), zeigen Heath und Luff (2000) am Beispiel einer Nachrichtenagentur auf, wie in der allgemeinen Hektik Aufmerksamkeit auf einzelne Meldungen gelenkt wird und sich die Journalisten wechselseitig ihrer Einschätzung bezüglich dieser potenziellen Nachricht versichern. Clayman und Reisner (1998) untersuchen einen konkreten, in der Medienforschung klassischen Gegenstand konversationsanalytisch, den Gatekeeper. Anhand von Tonbandaufzeichnungen der "editorial conferences" von insgesamt acht Zeitungsredaktionen wird „Gatekeeping in Action“ untersucht. Wie in der Konversationsanalyse üblich, wird nicht vom Gatekeeper als einer vorgefundenen personifizierten Realität gesprochen, sondern von „Gatekeeping“. Im Unterschied zu den vorliegenden Studien aus der Medienforschung, die sich vorzugsweise mit den Ergebnissen des Selektionsprozesses auseinander setzen, zeigen Clayman/Reisner den Prozess des Gatekeeping, also wie „newsworthiness“ während der Konferenzen erst hergestellt wird, wie also, um in der Sprache der Konversationsanalyse zu bleiben, ein „doing being newsworthy“ respektive ein „doing gatekeeping“ interaktiv überhaupt erzeugt wird.

\subsection{Rezeptionsanalysen}

$\mathrm{Zu}$ den ersten konversationsanalytischen Rezeptionsuntersuchungen sind die Untersuchungen von Ulmer/Bergmann (1993) und Keppler (1994) zu zählen. Sie hatten anhand von Tonbandaufzeichnungen von Familientischgesprächen aufgezeigt, wie mediale Inhalte ex post in den Familiengesprächen aufbereitet, rekonstruiert und re-inszeniert werden. Ulmer/Bergmann trennen kurze Medienverweise von längeren Medienrekonstruktionen. Medienrekonstruktionen - die Vergegenwärtigung von vergangener Medienerfahrung im Gespräch - können zum Beispiel als Kommemorationen oder auch als Re-Inszenierungen erfolgen. Keppler zeigt, welche Rolle Medienrekonstruktionen in der kommunikativen Vergemeinschaftung bei Tischgesprächen spielen. Medieninhalte 
„wirken“ nicht einfach, sie müssen „durch das Nadelöhr der alltäglichen Kommunikation" (Keppler 1994, 251).

Was die Rezeption massenmedialer Produkte während der Rezeptionssituation betrifft, hat ein interdisziplinäres, gesprächsanalytisch und konversationsanalytisch arbeitendes Forschungsprojekt unlängst Resultate vorgelegt. Diese Studien zeigen auf, wie sich natürliche Rezeptionsgemeinschaften in der Rezeptionssituation in situ massenmediale Inhalte kommunikativ aneignen (vgl. die Beiträge in Holly/Püschel/Bergmann 2001 sowie Klemm 2000 und Baldauf 2002, siehe aber auch schon Matthewson 1992). Im Kontext dieses Projekts sind neben gesprächsanalytischen auch konversationsanalytische Untersuchungen im engeren Sinn entstanden. So wird zum Beispiel aufgezeigt, dass Umschalten (Zappen), ein Zuschauerhandeln, das bislang vorwiegend anhand telemetrischer Daten oder über Befragungen untersucht wurde, mehr ist als ein mechanischer Selektionsvorgang. Vielmehr stellt es eine methodische Aktivität der Rezipienten dar, welche in der Rezeptionssituation selbst interaktiv ausgehandelt wird und zu bestimmten Mustern im gezappten Fernsehtext führt (vgl. Ayaß 2003b). Gerade für Rezeptionsstudien zeigt sich die enorme Stärke der konversationsanalytischen Methode und ihres spezifischen Wirklichkeitszugriffs. Denn indem sie sich nicht auf die Erinnerungen von Befragten verlassen muss und auch dem Problem der „Verzerrungen“ von Antworten hinsichtlich einer sozialen Erwünschtheit aus dem Weg gehen kann, erreicht sie einen Zugriff auf die natürliche Rezeptionssituation, der gängigen Verfahren der Rezeptionsforschung mit ihren entweder rekonstruktiven Erhebungsmethoden oder experimentellen Anordnungen verstellt ist.

\subsection{Produktanalysen}

Die mit Abstand meisten konversationsanalytischen Untersuchungen im Kontext medialer Kommunikation befassen sich mit der Analyse medialer Produkte. Unschwer lassen sich zwei Hauptakzente erkennen: Radiosendungen, insbesondere so genannte Call-InSendungen, und Fernsehnachrichten, darunter fast ausschließlich Nachrichteninterviews. So erratisch diese Schwerpunktsetzung auf den ersten Blick auch scheinen mag, folgt sie einer aus der Geschichte der Konversationsanalyse heraus nachvollziehbaren Logik.

In Radiosendungen sind die Produzenten und Rezipienten auf einen rein auditiven Kanal der Vermittlung angewiesen. Mithin wird mit dieser Art von Datenkorpus eine Tradition fortgesetzt, die mit der Wahl von Telefongesprächen als Gegenstand der frühen Konversationsanalyse ihren Ursprung hatte. Wie in Telefongesprächen auch, entfallen in Radiosendungen - zumindest für die Rezipienten - die visuellen Mitteilungsebenen, so dass sämtliche Ressourcen der Interpretation, die den „natürlichen“ Rezipienten zur Verfügung stehen, auch in den Tonbandmitschnitten für die Analyse enthalten sind. Mit dem Schwerpunkt auf Radio-Call-Ins innerhalb der konversationsanalytischen Untersuchung von Radiokommunikationen wird an einem klassischen Gegenstand der Konversationsanalyse festgehalten: die Analyse von Telefongesprächen (z. B. Liddicoat et al. 1992; Hutchby 1991; 1996a; 1996b; 1999; 2001). Die Vorliebe für das Radio und insbesondere für Hörertelefonate („Call-Ins“) innerhalb der Radiosendungen setzt damit im doppelten Sinn eine konversationsanalytische Tradition fort, insofern eine Art „natürlich reduzierte“ Kommunikationsform gewählt wird, in welcher der Konversationsanalyse an Interpretationsressourcen all das - und nur das - zur Verfügung steht, was auch die Rezipienten zur Interpretation in Anspruch nehmen können. Dies führt sogar dazu, dass in einer Analyse von einer Fernsebsendung der Schwerpunkt auf die Call-Ins der Zuschauer gelegt wird (Crows Analyse der Fernsehsendung „Good Sex“, 1986). 
Die Wahl des zweiten hauptsächlichen Gegenstands, Fernsehnachrichten, ist für die Medienforschung von besonderem Interesse. Fernsehnachrichten gehören international zu den meistbeforschten Sendeformen. Mit der Analyse speziell der Nachrichteninterviews hat die Konversationsanalyse jedoch ein Forschungsfeld betreten, das bislang in der internationalen Nachrichtenforschung eher wenig Beachtung fand. Auch hier ist unschwer zu erkennen, dass die Wahl dieses speziellen Gebietes seitens der Konversationsanalyse keineswegs zufällig erfolgt: Nachrichteninterviews bestehen fast ausschließlich aus Frage-Antwort-Sequenzen, Paarsequenzen also.

Die gegenwärtige Zusammenfassung klassifiziert die konversationsanalytischen Untersuchungen nach dem in der Medienforschung geläufigen, traditionell dreiteiligen Schema von Produkt-, Produktions- und Rezeptionsstudien. Es ist insgesamt für die konversationsanalytischen Untersuchungen jedoch bezeichnend, dass sie sich eben nicht konsequent in das traditionelle Schema einordnen lassen. ${ }^{12}$ Bevor im folgenden Abschnitt 4 die wesentlichen Resultate der Nachrichtenforschungen gesondert präsentiert werden, sollen daher in einem kurzen Überblick weitere Untersuchungen zu massenmedialer Kommunikation aus Konversationsanalyse und Ethnomethodologie genannt sein, die für die Medienforschung von Interesse sind.

Politische Rhetorik und Publikumshandeln: Schon als Klassiker kann Atkinsons „Our master's voices“ (1984) bezeichnet werden, eine Untersuchung, die die Techniken der Applaus-Elizitierung bei (englischen) Politikern aufzeigt. Atkinson zeigt, welche rhetorischen Techniken (Listenkonstruktionen, Kontrastpaare etc.) als „claptrap“ fungieren. Heritage/Greatbatch behandeln die politische Rhetorik von Parteitagen (1986). Mit dem Gegenstück dazu beschäftigt sich Clayman (1993a), dem „booing“, und Hutchby mit politischen Fernsehdiskussionen (1997). Bilmes (1999) untersucht die Struktur von Frage/Antwort-Sequenzen im Wahlkampf. Clayman/Heritage (2002a) zeigen nicht nur auf, dass angriffslustige Fragen seitens der Journalisten während der Pressekonferenzen zunehmen, sondern auch, worin diese spezielle Aggressivität besteht bzw. wie sie seitens der Journalisten hergestellt wird.

Schriftsprachlich entworfene oder "monologische“ Kommunikation: Auch wenn viele mediale Sendeformen auf interaktiven Konstellationen basieren, welche sie für die Konversationsanalyse so interessant machen, existieren einige Untersuchungen, die sich mit „monologischer" und/oder schriftsprachlich entworfener massenmedialer Kommunikation befassen, wie etwa Montgomerys (1986; 1991) Untersuchungen zu „DJ talk“.

Mediale Gattungen: Im deutschen Sprachraum entwickelte sich in Anschluss an die Gattungsanalysen von Alltagskommunikationen (Luckmann 1986; Bergmann/Luckmann 1995), welche ihrerseits auf der Konversationsanalyse aufbauen, eine spezielle Form von Produktanalyse, die mediale Formate als mediale Gattungen begreift und auf ihren Verfestigungscharakter hin untersucht. Unter Gattungen werden strukturelle „Lösungen“ für kommunikative „Probleme“ verstanden. Entsprechend sind mediale Gattungen Lösungen für ein entsprechend gelagertes Problem. Als Beispiele wären die Analysen über Werbespots von Ayaß (2002) und Knoblauch/Raab (2002) zu nennen so-

12 Dies wirft die Frage auf, inwiefern dann die gerade erfolgende Darstellung anhand dieses Schemas sinnvoll ist. Aus Sicht der Konversationsanalyse ist sie es sicherlich nicht (vgl. hierzu auch Abschnitt 5). Doch wie Äußerungen in Interaktionen ein „recipient design“, einen Rezipientenzuschnitt, aufweisen - und aufweisen müssen, um verständlich zu sein -, gilt ebensolches für wissenschaftliche Texte. 
wie Ayaß’ Analyse des „Worts zum Sonntag“ (1997) als einer medialen Gattung, deren „Problem“ darin besteht, dass sie einerseits von einem unchristlichen Zuschauer ausgeht, andererseits das Gattungswissen eben jener Zuschauer unterschätzt. Auch historisch neuere Formen verzeichnen einen Verfestigungscharakter: Schmidt (2000) diskutiert den gattungshaften Charakter von Chat-Kommunikation; Androutsopoulos/ Schmidt (2002) untersuchen äußere und innere Formen der SMS („short message system") als mediale Gattungen.

„Membership categories": Ebenfalls vorwiegend mit schriftlichem Material befasst sich eine spezielle Ausprägung der Ethnomethodologie, die Analyse von Kategorien und Kategorisierungen. Sie ist mit der Konversationsanalyse nur mehr verwandt, ist aber wie sie ethnomethodologischen Ursprungs und weist eine lange, ebenfalls auf Harvey Sacks zurückgehende Tradition auf. Sie ist im Englischen mit den analytischen Begriffen „MCD“ („membership categorization devices“) und „CBA“ („category bound activities“) bekannt geworden (vgl. umfassend Jayyusi 1984). „Category bound activities“ bestehen aus typischen Handlungen, die Kategorien zugewiesen werden bzw. von ihnen erwartet werden. „Membership categorization devices“ sind im Gegenzug interaktive Verfahren, mittels derer Personen einer Kategorie zugeschrieben werden. Sacks' Beispiel - aus einer Zeitungsmeldung - lautet: „In the last year and a half 26 Negroes were killed in the South, in unsolved murders“. Durch die Wahl der Begriffe (Personen, Ort) und die Begriffskonstellation wird ein Interpretationsfeld eröffnet, das einen rassistischen Hintergrund der Tat nahe legt (1992, Bd. 1, 577). Lees (1984) Analyse einer Schlagzeile („Girl guide aged 14 raped at Hell's Angels convention“) schließt direkt an Sacks' Überlegungen an. Er zeigt, mit welchen Präsuppositionen diese Überschrift arbeitet. Unmittelbar würde sie als „morally contrastive categories“ von „innocent victim“ and „evil-doers“ gelesen und direkt den „evil-doers“ die Tat unterstellt, obwohl in der Schlagzeile davon zunächst nicht die Rede ist $(1984,70)$. Zentral dafür verantwortlich sind eben die „category bound activities“, die z. B. der Kategorie „Hell’s Angels“ als typischem „evil-doer“ (im Gegensatz zu einem jungen Girl-Scout, das unmittelbar der Kategorie „innocent victim“ zugewiesen wird) zugeschrieben würden: „Hell’s Angels do that sort of thing “ $(1984,70 / 71) \cdot{ }^{13}$

\section{Das Nachrichteninterview als kollaborativer Herstellungsprozess}

Die mit Abstand meisten konversationsanalytischen Medienstudien konzentrieren sich auf Nachrichteninterviews. Für die Medienforschung sind diese Untersuchungen von besonderem Interesse, da sie eine Lücke füllen. Die Darstellung konzentriert sich daher im Folgenden ausführlicher auf die wesentlichen Resultate dieser Untersuchungen. Angesichts der Fülle der Untersuchungen zu Nachrichteninterviews ${ }^{14}$ wird vorwiegend auf drei „klassische“ frühe Texte von Greatbatch, Heritage und Clayman Bezug genommen (4.1) sowie ein Themenschwerpunkt vorgestellt: das Sonderheft von „Research on Language and Social Interaction“ zum „Rather/Bush-Encounter“, einem spektakulären

13 Für weitere Beispiele zu Analysen von „membership categories“ in Zeitungsberichten siehe Schenkein 1979, sowie eine Reihe der Beiträge im von Jalbert 1999 herausgegebenen Sammelband: Stetson 1999, Hester/Fitzgerald 1999 und Eglin/Hester 1999.

14 Allein mit Nachrichteninterviews befassen sich (ohne Anspruch auf Vollständigkeit): Clayman 1988, Clayman 1991, Clayman 1992, Clayman 1993b, Clayman 2001, Clayman/Whalen 1988/1989, Clayman/Heritage 2002a, Clayman/Heritage 2002b, Corner 1991, Ekström 2001, Greatbatch 1986a, Greatbatch 1986b, Greatbatch 1992, Harris 1986, Harris 1991, Heritage 1985, 
Nachrichteninterview aus dem Jahr 1988, in dem sich Moderator und Gast gegenseitig niederschrieen (4.2).

\section{1 „Doing Interviewing“: Das Nachrichteninterview als kollaborative Produktion}

Viele der Untersuchungen eröffnen zunächst mit einem Vergleich zwischen den evidenten strukturellen Besonderheiten der Nachrichteninterviews mit alltäglicher Faceto-Face-Kommunikation. Sie stellen zunächst lapidar fest, dass Nachrichteninterviews sich dadurch auszeichnen, dass die Verteilung der Redezüge durch institutionelle Restriktionen vorab auf ein Frage-Antwort-Format festgelegt sind, wobei dem Interviewer die Rolle des Fragenden, dem Interviewten die Rolle des Antwortenden zukommt. Hutchby (1991) zeigt die Differenzen mit einem Vergleich zwischen Telefongesprächen im Alltag und Phone-Ins in Medien (Hörfunk) auf. Diese zunächst eher wenig überraschende und zudem auch keineswegs allein auf Nachrichteninterviews zutreffende Feststellung wird jedoch durch weitere Beobachtungen revidiert, an denen aufgezeigt wird, wie die Beteiligten an der Interviewsituation das Interview als Interview hervorbringen. So starr die Redezugverteilung auf das Frage/Antwort-Schema festgelegt sein mag, wird doch zum Beispiel sichtbar, dass die Interviewer nicht nur Fragen stellen, und die Interviewten oft auch keine Antworten geben (Greatbatch 1988). Dennoch produzieren beide Beteiligten Äußerungsformate, die zumindest minimal als Fragen resp. Antworten erkennbar sind und gelten können, i.e. die Interagierenden orientieren sich wechselseitig an dieser Erwartung. Abweichungen werden entsprechend mittels Reparaturen sanktioniert. Interviewer zum Beispiel formulieren häufig auch inhaltliche Aussagen, doch werden diese meist direkt von Fragen gefolgt; Interviewte im Gegenzug behandeln diese Aussagen als „preliminaries“ und warten mit ihren Antworten, bis eine erkennbare Frage formuliert wurde (Greatbatch 1988, 407-410). Indem sich die Interviewten solange mit ihren Äußerungen zurückhalten, bis sie eine Frage oder wenigstens eine Art Frage erkennen können, behandeln sie die Äußerung des Interviewers als unvollendet. Ein kurzer Auszug aus einem der für die Konversationsanalyse typischen Transkripte soll dies veranschaulichen.

(Bei dem interviewten Politiker handelte es sich um den südafrikanischen Botschafter in den USA; Südafrika hatte gerade den Ausnahmezustand verhängt; zitiert nach Clayman (1988, 482). IR ist der Interviewer, IE der Interviewte):

IR: Finally Mister Ambassador as you know the critics say that the purpose of the state of emergency the real purpose of the state of emergency is to suppress political dissent. those who are opposed to the apartheid government of South Africa. Is that so

IE: I would have to take issue with that premise because (...)

Der Interviewte (IE) hält sich während der Äußerung des Interviewers solange zurück, bis die Frage erkennbar ist. Clayman betont daher die interaktive Herstellung dieser Formate und die kollaborative Produktion der Fragen und Antworten (1988, 480):

"The questioning character of the resulting turn must therefore be regarded as the interactional achievement of both interviewers and interviewees, who collaborate to produce turns that are structured in this way."

Heritage/Clayman/Zimmerman 1988, Heritage/Greatbatch 1991, Heritage/Roth 1995, Myers 2000, Nofsinger 1988/1989, Rama Martinez 1993, Roth 1998, Schegloff 1988/1989. 
Interviewer produzieren auch häufig inhaltliche Statements, die nicht immer von Fragen gefolgt werden. Wie Clayman zeigen kann, enthalten diese Äußerungsformate häufig Zitate Dritter in meist indirekter Rede. Auch im oben zitierten Auszug ist ein solches, hier anonym gehaltenes, Zitat enthalten („the critics say“). Mittels dieser Redewiedergaben wird aber die Meinung einer dritten Person kundgegeben, bezüglich der nun erwartet wird, dass sich der Interviewte dazu äußert, was diese in der Regel auch tun. Die Journalisten lösen mit diesem Äußerungsformat eine komplexe Aufgabe: Sie drängen mittels zugespitzter Äußerungen die Interviewten zu Antworten und stellen sich andererseits als diejenigen dar, die nicht selbst Autoren dieser Äußerungen sind. Im Rahmen der Nachrichteninterviews ist dies eine von mehreren seitens der Interviewer eingesetzten Techniken, Neutralität herzustellen oder zu demonstrieren. Wiederum orientieren sich die Interviewten an diesem Verfahren und behandeln die Äußerung nicht als Meinung des Journalisten, sondern als Bericht. Interviewer enthalten sich weitestgehend aller wertenden, zustimmenden oder ablehnenden Äußerungen. Sie produzieren ihre Äußerungen in einem Format, das signalisiert, dass nicht sie selbst diese Meinungen vertreten, sondern quasi als Anwalt der Sache auftreten. Clayman resümiert:

„Accordingly, the successful achievement of neutrality is an interactional matter in which interviewees play a constitutive role and which the parties to an interview jointly accomplish as an observable state of affairs." $(1988,489)$

Den Journalisten gelingt es dadurch, einerseits zwar kontrovers zu fragen, andererseits jedoch ihre Neutralität zu markieren. Diese Neutralität wird auch mit weiteren Mitteln erzeugt. Wie vor allem Heritage zeigt, verzichten Interviewer nicht nur einfach auf alle wertenden Äußerungen, sondern enthalten sich generell der Rolle eines primären Rezipienten der vom Interviewten produzierten Antworten. So fehlen zum Beispiel die sonst so typischen Hörersignale:

"Thus in eliciting reports, while avoiding the responsive displays of a report recipient, interviewers adopt the neutral stance of one whose task is to assist in the production of talk for overhearers." $(1985,115)$

Den Begriff des „overhearer“ leiht Heritage bei Goffman, der in seinem Aufsatz „Footing“ (1981a) gezeigt hatte, dass die traditionelle Aufteilung von Beteiligten an einer Interaktion mit den Begriffen Sprecher und Hörer unzureichend ist. Wenn die Interviewer nicht als Autor (,author“) einer Äußerung auftreten, sondern als ein Sprachrohr (,animator"), verschieben sie das Footing ihrer Äußerungen. Indem sie sich aller typischen rezipientenspezifischen Äußerungen wie Hörersignale, „news receipts“ etc. enthalten, etablieren sie sich selbst als der nicht primäre Adressat im Sinne eines einfachen „hearer“. Es ist vielmehr ein „overhearer“, für den Journalisten Fragen stellen und interviewte Politiker Antworten produzieren. An dieser abwesenden „overhearing audience“ dem Fernsehpublikum - orientieren sich Interviewer und Interviewte gleichermaßen. Diese Orientierung ist eine kollaborative Herstellensleistung beider Parteien, die entscheidend zum „doing interviewing“ beiträgt.

\section{2 „Undoing Interviewing “: Der Wortwechsel zwischen Dan Rather und George Bush}

Mit einem speziellen Interview befasst sich eine „Special Section“ von „Research on Language and Social Interaction“ (1988/89): der Begegnung zwischen dem Nachrichtensprecher der amerikanischen Fernsehgesellschaft CBS Dan Rather und dem (damaligen) Vize-Präsidenten und Präsidentschaftskandidaten der USA George Bush sen. am 25. Januar 1988 in den „CBS Evening News“, ein Interview, das in eine lautstarke Auseinandersetzung eskalierte, in den USA großes Aufsehen erregte und zum Beispiel in der 
„Time“ als „video high noon“ bezeichnet wurde (zitiert nach Clayman/Whalen 1988/89, 242) ${ }^{15}$ Hier waren die unausgesprochenen Regeln des Interviews verletzt worden. Genau darum ist es, als „abweichender Fall“, besonders interessant. Abweichende Fälle spielen in der Konversationsanalyse für die Interpretation eine große Rolle. Sie werden als methodische Ressource genutzt. Anders als in vielen anderen methodischen Verfahren, werden sie nicht als Ausreißer aus dem Material exkludiert, sondern vielmehr systematisch einbezogen, um nicht nur anhand der Abweichung die Störung der Ordnung, sondern vielmehr die Orientierung der Interagierenden an dieser Störung und damit eben an der zugrundeliegenden Ordnung aufzuzeigen.

Das Interview begann wie ein „normales“ Interview: Rather fragte und Bush antwortete, und die Beteiligten, Bush und Rather, produzierten zunächst die oben beschriebenen interviewtypischen Sequenzen. So eröffnete Rather mit einem längeren Statement, an das sich eine Frage anschloss, Bush im Gegenzug wartete, ohne seinerseits Anstalten zu machen, vorzeitig eine Antwort zu produzieren, auf eine als Frage erkennbare Äußerung („why is...“), an deren Anschluss er direkt eine als Antwort auf die Frage erkennbare Äußerung produzierte („because...“). Clayman/Whalen (1988/89) und Schegloff (1988/89) zeigen nun, wo und wie das Interview als Interview aus den Fugen geriet und zu einer Verbalfehde wurde. Es war, auch wenn in den Medien später in erster Linie Rathers Verhalten diskutiert und kritisiert wurde, zunächst vor allem Bush, der begann, den Rahmen des Interviews zu verlassen. Dennoch, dies heben die Analysen einhellig hervor, war die Transformation des Geschehens „from interview to confrontation" (Schegloff) eine interaktive Herstellensleistung:

„Whatever prior agendas or predispositions there may have been, the actual course of the encounter must be treated as an emergent and fundamentally interactional achievement. Accordingly, if the encounter's trajectory differed from most ordinary news interviews, we want to arrive at a technical understanding of what those differences consisted of, and how they were methodically produced." (Clayman/Whalen 1988/89, 243)

Exakt diese methodischen Prozeduren werden in den Analysen en détail beschrieben: Bush begann zum Beispiel, sich während der den Fragen als „preliminaries“ vorgeschalteten Statements nicht wie ein typischer Interviewter zu verhalten, sondern versuchte, Rather den „turn“ abzunehmen, indem er ihn unterbrach und Antworten produzierte oder zumindest zu produzieren versuchte, bevor die Fragen auch nur gestellt waren. Dies führte zu Kämpfen ums Rederecht und teilweise auch dazu, dass Rather auf sein Recht zu fragen insistierte („Let me ask the question if I may first“) und damit seinerseits Bush unterbrach. ${ }^{16}$ Rather trug damit seinen Teil dazu bei, dass das Interview mehr und mehr aus gleichzeitiger, sich überlappender Rede bestand, bei der nicht einer der beiden Kontrahenten dem anderen den „floor“ überließ, sondern es zu regelrechten Kämpfen um das Rederecht kam, die den Eindruck eines teils kaum verständlichen „shouting match“verursachten.

15 Das „Rather-Bush-Encounter“, auf das die „Special Section“ Bezug nimmt, ist dort im Anhang des Heftes, zusammen mit der vorausgegangenen Filmeinspielung, auf die im Interview mehrfach Bezug genommen wird, vollständig transkribiert. In den USA ist es als Kaufvideo erhältlich. Die Videosequenz zirkuliert auch im Internet und kann zum Beispiel unter http://secure.mediaresearch.org/news/rathervideos.html\#1 (Zugriff vom 19.9.2003) angesehen werden.

16 Für die vorliegenden illustrativen Zwecke wurden die Transkriptionen der zitierten Auszüge vereinfacht. 
Die Analysen zeigen, wie Schritt für Schritt beide Parteien Äußerungen produzierten, die für ein Interview nicht typisch sind. Bush beschwerte sich angesichts Rathers wiederholter Nachfragen zu Bushs Wissen in der Iran-Contra-Affäre mehrfach über das Nichteinhalten von Absprachen („I'm asking for fair play and I thought I was here to talk abvout my views on education or on getting this deficit down“, „You invited me to come here to talk about I thought the whole record“ etc.). Insbesondere Bushs persönliche Angriffe auf den Moderator und das Programm führen zu einem Wandel der stattfindenden kommunikativen Form. Bushs Parade gegen Rather - „How would you like that if I judged your career by those seven minutes when you walked off the set in New York. (...) Would you like that?" - spielte auf ein Ereignis an, als Rather im September 1987 aus Protest gegen eine Verschiebung seines Nachrichtenprogramms wegen einer Tennisübertragung das Studio verließ und CBS sieben Minuten lang einen leeren Bildschirm bescherte. Vor allem aber ist es neben dem im Rahmen eines Interviews unüblichen Angriff auf die Person des Interviewers eine nominelle Umkehr der Rollen des Fragenden und des Befragten. Plötzlich war der Moderator in der für ein Nachrichteninterview völlig ungewöhnlichen Situation des Befragten und Bedrängten. (Entsprechend konsterniert reagierte auch Rather.) Spätestens an dieser Stelle hatte, was geschah, mit einem Interview nichts mehr zu tun, oder, wie es Schegloff formuliert: „(...) doing interview has broken down." $(1988 / 89,227)$

Weitere Angriffe Bushs folgten: „I have respect for you, but I don't have respect for what you are doing here tonight"; "There is nothing new here. I thought this was a news program“. Rather seinerseits ließ sich provozieren. Er beschuldigte Bush („You’ve made us hypocrites in the face of the world“) und beendete das Interview jählings, indem er Bush eine Frage stellte und, noch während Bush antwortete, Bush mittels eines „I gather that the answer is no" unterbrach und das Interview beendete.

Die Analysen zeigen, dass ein Interview nicht einfach ein gegebener Rahmen ist, innerhalb dessen alles, was stattfindet, „interviewhaft“ ist:

"An interview is thus a framework of accountability that inhabits action and is sustained through it, rather than a preconstituted situation that exists outside of action or determines it." (Clayman/Whalen 1988/89, 267)

Ein Interview ist eine sukzessive Leistung aller Beteiligten, in der das „Interviewhafte“ des Interviews durch ein „doing interview“ hergestellt wird - oder aber eben auch ein „undoing interviewing“ betrieben wird, wie im Beispiel des Rather-Bush-Zwischenfalls.

\section{Kritik und Ausblick}

Über die bislang von der Konversationsanalyse bearbeiteten Bereiche der Massenmedien hinaus gibt es eine Fülle von weiteren möglichen Untersuchungsobjekten. Mit der Fokussierung auf Nachrichteninterviews haben sich die bisherigen Untersuchungen einen ausgesprochen engen Fokus gegeben. Wie gezeigt, entstammt dieses Interesse an Frage-Antwort-Sequenzen der Tradition der Konversationsanalyse und ihrer intensiven Beachtung der geordneten Strukturen von Paarsequenzen. Dabei ließe sich zum Beispiel das von Clayman aufgezeigte „Displaying Neutrality“ der fragenden Journalisten erweitern in ein „Doing neutrality“ oder ein „Doing objectivity“ der Nachrichtenberichterstattung insgesamt. Gaye Tuchman hatte ja schon (1972; 1973) Objektivität in Nachrichtenherstellung als „strategisches Ritual“ und als ein „Doing ,objective“ TV news film" beschrieben. Darüber hinaus wäre gerade bei Interviews, bei denen ja höchster Wert auf Live-Sendung gelegt wird, der Aspekt der Herstellung von Authentizität 
und Aktualität zu beachten. Konversationsanalytisch kann man hier von einer Herstellung von „here-ness“ und „now-ness“ sprechen.

Darüber hinaus scheint ein Grundgedanke der Konversationsanalyse für die Medienforschung äußerst viel versprechend: Die Analyse von Störungen und Zusammenbrüchen der Ordnung. Garfinkel hatte in seinen berühmten Krisenexperimenten aufgezeigt, wie dünn der Boden der sozialen Wirklichkeit ist, wie brüchig die gemeinsamen Konstruktionen sind. Brüche und Krisen zeigen auf, was Garfinkel das „seen but unnoticed“ (1967b, 41) nennt. Gerade die Massenmedien, bei all ihrem Perfektionsanspruch, produzieren und transportieren solche Brüche allenthalben und liefern sie frei Haus, wie die Analyse des Rather-Bush-Zwischenfalls zeigte. Um beim Standardbeispiel Nachrichten zu bleiben: Zu den Brüchen und Störungen sozialer Ordnung zählt sowohl Dagmar Berghoffs Versprecher inklusive Lachanfall während der Tagesschau, als auch die Berichterstattung zum 11. September, als im amerikanischen Fernsehen in Live-Sendungen erfahrene und routinierte Journalisten die Fassung verloren. Unter den klassischen Pannen sind insbesondere jene Situationen interessant, in denen es zu nicht ganz freiwilligen Einblicken auf die Hinterbühne kommt, wenn etwa zum Beispiel zu Korrespondenten geschaltet wird, die sich noch nicht auf Sendung wähnen oder in Studios Regler geöffnet sind, die die Anwesenden für geschlossen halten. Was für die Medien eine „Panne“ ist, ist für die Konversationsanalyse vielmehr eine systematische Ressource. ${ }^{17}$

Die bisherigen Analysen erwecken den Eindruck, es seien in erster Linie Produktanalysen für die Konversationsanalyse interessant, darunter insbesondere interaktive Konstellationen wie eben das Nachrichteninterview. Neben Nachrichteninterviews existieren in Massenmedien jedoch eine Fülle weiterer interaktiver Situationen, die sich als empirischer Gegenstand der Konversationsanalyse geradezu anbieten, da sie Wirklichkeitskonstruktionen par excellence produzieren, etwa Wahlabende oder Volksmusiksendungen. Für solche Sendungen gelten entsprechend andere „doings“ - „doing suspense“, „doing being funny“, „doing being a ,Volksmusiksendung“" etc. Jenseits der interaktiven Situationen bieten sich aber auch „monologische“ und/oder schriftsprachlich konstituierte Kommunikationen wie Kommentare oder Wettervorhersagen an. Ein ganz neues Forschungsfeld wären fiktive Darstellungsformen, die auf den ersten Blick als Material für eine Konversationsanalyse untauglich zu sein scheinen (vgl. jedoch für (ethno-)methodologische und methodische Überlegungen Jayyusi 1988 und McHoul 1987).

Die Schritte, die die konversationsanalytische Medienforschung in den kommenden Jahren zu gehen hat, betreffen zum einen ihre Öffnung zur Medienforschung (1) und zum anderen ihre Sensibilisierung bezüglich der Medialität ihres Materials (2). Zum ersten Punkt: Das Problem, das viele der bisherigen konversationsanalytischen Untersuchungen haben, ist, dass sie den Anschluss an die Medienforschung - von wenigen personellen Ausnahmen abgesehen - nicht finden, da sie ihn selbst nicht suchen. Dies liegt auch daran, dass sich die Konversationsanalyse medientheoretischen Fragen in vielen Punkten systematisch versperrt. Zunächst sind ihr die in der Medienforschung geläufigen Unterscheidungen von „Sender“ und „Empfänger“ (oder neuerdings Produzent und Rezipient) schlicht fremd. Auch die gängige Differenzierung der Medienforschung

17 Eine vergnügliche Sammlung solcher Pannen findet sich unter http://www.radiopannen.de. Zu einer Analyse von Versprechern im Radio und ihrer Bewältigung durch die Moderatoren siehe schon Goffman (1981b). 
in Produkt-, Produktions- und Rezeptionsstudien ist aus Sicht der Konversationsanalyse genau genommen nicht sinnig. Denn mit der Frage, wie denn soziale Ordnung seitens der Interagierenden hergestellt wird, hat man - mit dem Begriff des „doing“ - schon vorweg eine „Produktions“-Perspektive eingenommen, wenn auch in einem gänzlich anderen Sinne als in der Medienforschung üblich: die Perspektive derer, die die soziale Wirklichkeit herstellen und soziale Ordnung erzeugen. Die methodische Herangehensweise der Konversationsanalyse steht daher den üblichen Fragestellungen der Medienwissenschaften teils diametral gegenüber. Denn während in gängigen Untersuchungen zum Beispiel Objektivität als nahezu ontologisch gegeben behandelt wird und nur mehr gemessen wird, 'wie objektiv' zum Beispiel Nachrichten sind, würde die Konversationsanalyse im Gegenzug fragen, wie denn diese Objektivität überhaupt erst hergestellt wird.

Dabei bestehen, bei allen Gegensätzen, inhaltliche Anknüpfungspunkte gerade dort, wo man sie am wenigsten vermutet. Zwei exemplarische Beispiele aus aktuelleren Untersuchungen ließen sich nennen: Fürsichs (1994) Analyse der Szenerie des Studios und des visuellen Arrangements in Nachrichtensendungen liefert eine (im irritierenden Befremden der Betrachtungsweise in manchen Punkten nahezu ethnomethodologische) Beschreibung des rituellen Charakters von Fernsehnachrichten. Miller und McHoul (1998) finden verblüffende Überschneidungen zwischen Ethnomethodologie und Cultural Studies. Denn beide Ansätze untersuchen die „everyday practices“ alltäglich Interagierender - wenn auch die Cultural Studies ein Faible für das Spektakuläre, die Konversationsanalyse hingegen eher für das Unscheinbare, das „seen but unnoticed“, hat.

Der zweite Punkt, in dem für die konversationsanalytische Medienforschung noch viel Spielraum besteht, betrifft eine konsequente Medialisierung des Materials und der Analyse. ${ }^{18}$ Gegenwärtig macht die Konversationsanalyse ihr Material und ihre Analysen für eine Medienforschung nicht ausreichend attraktiv, da sie den medialen Charakter des Materials nicht hinlänglich berücksichtigt. Bezeichnend für die oben zitierten Untersuchungen über massenmediale Kommunikation ist, dass in Transkripten und damit auch in den Analysen auf die bildliche Mitteilungsebene gleich vollständig verzichtet wird. Einstellungsgröße oder -wechsel, Schnitte, Kameraperspektive, Einblendungen wie „Inserts“, die „Fliege“ in der Ecke (das kleine Senderlogo), die bildlichen (BlueScreen oder neue virtuelle) Techniken oder Studio-Hintergründe - all diese typischen Darstellungsformen des Fernsehens sucht man in den zitierten Untersuchungen vergebens. Auch visuelle Elemente wie Blickkontakt oder -richtung, Position der Hände, Gestik, Mimik etc., Kopfschütteln, Nicken - in anderen konversationsanalytischen Untersuchungen, die mit Videoaufzeichnungen von Alltagssituationen arbeiten (bei Meier 1997, Goodwin 1981 oder Heath 1986 und vor allem in den neuen Untersuchungen der CSCW), sehr wohl en détail notiert - fehlen in Transkripten und auch in den Analysen ausgerechnet der konversationsanalytischen Medienstudien. Damit verschenken aber die genannten Untersuchungen bislang das, was streng genommen die Qualität ethnomethodologischer und konversationsanalytischer Analysen erst ausmacht, nämlich die Antwort auf die Frage: Was genau macht ein Fernsehnachrichteninterview zum Fernsehnachrichteninterview? Dabei existieren, gerade auch aus filmanalytischen Untersuchungen, verschiedene Modelle für eine Transkription audiovisuellen filmischen Mate-

$18 \mathrm{Zu}$ einer methodischen Kritik an der Konversationsanalyse in genau diesem Aspekt siehe Ayaß $2003 a$. 
rials sowie Begrifflichkeiten zur Bezeichnung, so dass hier das Rad nicht oder nicht gänzlich neu erfunden werden müsste.

Es ist jedoch derzeit erkennbar, dass sich die Praxis in den konversationsanalytischen Medienuntersuchungen unter dem Einfluss und dem Vorbild der innovativen Studien aus den CSCW, z. B. der genannten Weg weisenden Arbeiten von Suchman, Goodwin oder Heath, welche konsequent die Medialität des Untersuchungsgegenstands berücksichtigen und in die Analyse, auch in die Darstellung, einfließen lassen, ändert. An einer konsequenten Medialisierung ihres Materials und ihrer Analysen kommen auch die konversationsanalytischen Untersuchungen massenmedialer Kommunikation künftig nicht vorbei. Sie folgen damit, wenn auch mit Verspätung, der ethnomethodologischen Prämisse des „unique adequacy requirement“ (Garfinkel/Wieder 1992), demzufolge jede ethnomethodologische Untersuchung sich in ihrer Methode dem Material anzupassen hat.

Daher ist es für die Medienforschung ein guter Zeitpunkt, die empirischen Resultate der Konversationsanalyse zur Kenntnis zu nehmen und ihre weitere Entwicklung kritisch zu beobachten.

\section{Literatur}

Androutsopoulos, Jannis/Gurly Schmidt (2002), SMS-Kommunikation: Ethnografische Gattungsanalyse am Beispiel einer Kleingruppe, in: Zeitschrift für Angewandte Linguistik, Vol. 36, 49-80.

Atkinson, Max (1984), Our masters' voices. The language and body language of politics. London: Routledge.

Ayaß, Ruth (1997), „Das Wort zum Sonntag“. Fallstudie einer kirchlichen Sendereihe. Stuttgart: Kohlhammer.

Ayaß, Ruth (2002), Zwischen Innovation und Repetition. Der Fernsehwerbespot als mediale Gattung, in: Willems, Herbert (Hrsg.), Die Gesellschaft der Werbung. Kontexte und Texte. Produktionen und Rezeptionen. Entwicklungen und Perspektiven. Opladen: Westdeutscher Verlag, 155-171.

Ayaß, Ruth (2003a), Conversation Analysis' studies on mass media materials. What a consequential medialization of transcription can do for analysis. Universität Bielefeld, Manuskript.

Ayaß, Ruth (2003b), Zur sozialen Konstitution des Umschaltens (Zappens) in der Rezeptionssituation. Universität Bielefeld, Manuskript.

Baldauf, Heike (2002), Knappes Sprechen. Tübingen: Niemeyer.

Bergmann, Jörg (1988), Ethnomethodologie und Konversationsanalyse. 1: Ethnomethodologie: Untersuchungen zur methodischen Erzeugung von sozialer Wirklichkeit im alltäglichen Handeln; 2: Der Untersuchungsansatz der ethnomethodologischen Konversationsanalyse; 3: Organisationsprinzipien der sozialen Interaktion: Objekte der Konversationsanalyse. Kurseinheiten der Fernuniversität Hagen.

Bergmann, Jörg (2000a), Ethnomethodologie, in: Flick, Uwe/Ernst von Kardorff/Ines Steinke (Hrsg.), Qualitative Sozialforschung. Ein Handbuch. Reinbek: Rowohlt, 118-135.

Bergmann, Jörg (2000b), Harold Garfinkel und Harvey Sacks, in: Flick, Uwe/Ernst von Kardorff/Ines Steinke (Hrsg.), Qualitative Sozialforschung. Ein Handbuch. Reinbek: Rowohlt, $51-62$.

Bergmann, Jörg (2000c), Konversationsanalyse, in: Flick, Uwe/Ernst von Kardorff/Ines Steinke (Hrsg.), Qualitative Sozialforschung. Ein Handbuch. Reinbek: Rowohlt, 524-537.

Bergmann, Jörg/Thomas Luckmann (1995), Reconstructive genres of everyday communication, in: Quasthoff, Uta M. (Hrsg.), Aspects of oral communication. Berlin/New York: de Gruyter, 289-304.

Billig, Michael (1999a), Whose terms? Whose ordinariness? Rhetoric and ideology in Conversation Analysis, in: Discourse and Society, Vol. 10, Nr. 4, 543-582. 
Billig, Michael (1999b), Conversation Analysis and the claims of naivety, in: Discourse and Society, Vol. 10, Nr. 4, 572-576.

Bilmes, Jack (1999), Questions, answers, and the organization of talk in the 1992 vice presidential debate: Fundamental considerations, in: Research on Language and Social Interaction, Vol. 32, Nr. 3, 213-242.

Button, Graham (Hrsg.) (1993), Technology in working order. Studies of work, interaction, and technology. London: Routledge.

Churchill, Lindsey (1971), Ethnomethodology and measurement, in: Social Forces, Vol. 50, 183-191.

Clayman, Steven (1988), Displaying neutrality in television news interviews, in: Social Problems, Vol. 35, Nr. 4, 474-492.

Clayman, Steven (1991), News interview openings: Aspects on sequential organization, in: Scannell, Paddy (Hrsg.), Broadcast talk. London: Sage, 48-75.

Clayman, Steven (1992), Footing in the achievement of neutrality: The case of news-interviews discourse, in: Drew, Paul/John Heritage (Hrsg.), Talk at work. Interaction in institutional settings. Cambridge: Cambridge University Press, 163-198.

Clayman, Steven (1993a), Booing: The anatomy of a disaffiliative response, in: American Sociological Review, Vol. 58, 110-130.

Clayman, Steven (1993b), Reformulating the question: A device for answering/not answering questions in news interviews and press conferences, in: Text, Vol. 13, Nr. 2, 159-188.

Clayman, Steven (2001), Answers and evasions, in: Language in Society, Vol. 30, Nr. 3, 403-442.

Clayman, Steven E./Ann Reisner (1998), Gatekeeping in action: Editorial conferences and assessments of newsworthiness, in: American Sociological Review, Vol. 63, 178-199.

Clayman, Steven E./Jack Whalen (1988/1989), When the medium becomes the message. The case of the Rather-Bush encounter, in: Research on Language and Social Interaction, Vol. 22, 241-272.

Clayman, Steven/John Heritage (2002a), Questioning presidents. Journalistic deference and adversialness in the press conferences of U.S. presidents Eisenhower and Reagan, in: Journal of Communication, Vol. 52, Nr. 4, 749-775.

Clayman, Steven/John Heritage (2002b), The news interview. Journalists and public figures on the air. Cambridge: Cambridge University Press.

Corner, John (1991), The interview as social encounter, in: Scannell, Paddy (Hrsg.), Broadcast talk. London: Sage, 31-47.

Crow, Bryan K. (1986), Conversational pragmatics in television talk: The discourse of „Good Sex“, in: Media, Culture \& Society (Themenheft: „Broadcast talk“, ed. Paddy Scannell), Vol. 8, Nr. 4, 457-484.

Deppermann, Arnulf (1999), Gespräche analysieren. Eine Einführung in konversationsanalytische Methoden. Opladen: Leske und Budrich.

Egbert, Maria (1996), Context-sensitivity in conversation analysis. Eye-gaze and the German repair initiator „Bitte“, in: Language in Society, Vol. 25, 587-612.

Eglin, Peter/Stephen Hester (1999), Moral order and the Montreal massacre, in: Jalbert, Paul L. (Hrsg.), Media Studies: Ethnomethodological approaches. Boston: University Press of America, 195-230.

Ekström, Mats (2001), Politicians interviewed on television news, in: Discourse and Society, Vol. 12 , Nr. 5, 563-584.

Fürsich, Elfriede (1994), Fernsehnachrichten als Ritual. Ein neuer Ansatz zur Interpretation, in: Publizistik, Vol. 39, Nr. 1, 27-57.

Garfinkel, Harold (1967a), Passing and the managed achievement of sex status in an „intersexed“ person (part 1), in: ders., Studies in ethnomethodology. Englewood/Cliffs, NJ: Prentice Hall, $116-185$.

Garfinkel, Harold (1967b), Studies in ethnomethodology. Englewood/Cliffs, NJ: Prentice Hall.

Garfinkel, Harold/D. Lawrence Wieder (1992), Two incommensurable, asymmetrically alternate technologies of social analysis, in: Watson, Graham/Robert M. Seiler (Hrsg.), Text in context. Contributions to ethnomethodology. Newbury Park, CA: Sage, 175-206. 
Goffman, Erving (1981a), Footing, in: ders., Forms of talk. Philadelphia: University of Pennsylvania Press, 124-159 (orig. in: Semiotica, 1979, Vol. 25, 1-19).

Goffman, Erving (1981b), Radio talk. A study of the ways of our errors, in: ders., Forms of talk. Philadelphia: University of Pennsylvania Press, 197-327.

Goll, Michaela (2002), Arbeiten im Netz. Kommunikationsstrukturen, Arbeitsabläufe, Wissensmanagement. Opladen: Westdeutscher Verlag.

Goodwin, Charles (1981), Conversational organization. Interaction between speakers and hearers. New York: Academic Press.

Goodwin, Charles (1996), Transparent vision, in: Ochs, Elinor/Emanuel A. Schegloff/Sandra A. Thompson (Hrsg.), Interaction and grammar. Cambridge: Cambridge University Press, 370-404.

Goodwin, Charles/Marjorie H. Goodwin (1996), Seeing as a situated activity: Formulating planes, in: Engeström, Yrjö/David Middleton (Hrsg.), Cognition and communication at work. Cambridge: Cambridge University Press, 61-95.

Greatbatch, David (1986a), Aspects of topical organization in news interviews. The use of agendashifting procedures by interviewees, in: Media, Culture \& Society (Themenheft: Broadcast Talk, ed. Paddy Scannell), Vol. 8, Nr. 4, 441-455.

Greatbatch, David (1986b), Some standard uses of supplementary questions in news interviews, in: Wilson, John/Bryan K. Crow (Hrsg.), Belfast Working Papers on Language and Linguistics, Vol. 8. Jordanstown: University of Ulster, 86-123.

Greatbatch, David (1988), A turn-taking system for British news interviews, in: Language in Society, Vol. 17, 401-430.

Greatbatch, David (1992), On the management of disagreement between news interviewees, in: Drew, Paul/John Heritage (Hrsg.), Talk at work. Interaction in institutional settings. Cambridge: Cambridge University Press, 268-301.

Günthner, Susanne (1993), Diskursstrategien in der interkulturellen Kommunikation: Analysen deutsch-chinesischer Gespräche. Tübingen: Niemeyer.

Harris, Sandra (1986), Interviewers' questions in broadcast interviews, in: Wilson, John/Bryan K. Crow (Hrsg.), Belfast Working Papers on Language and Linguistics, Vol. 8. Jordanstown: University of Ulster, 50-85.

Harris, Sandra (1991), Evasive action: How politicians respond to questions in political interviews, in: Scannell, Paddy (Hrsg.), Broadcast talk. London: Sage, 76-99.

Heath, Christian (1986), Body movement and speech in medical interaction. Cambridge: Cambridge University Press.

Heath, Christian/Jon Hindmarsh/Paul Luff (1999), Interaction in isolation: The dislocated world of the London underground train driver, in: Sociology, Vol. 33, Nr. 3, 555-575.

Heath, Christian/Paul Luff (1993), Disembodied conduct. Interactional asymmetries in video-mediated communication, in: Button, Graham (Hrsg.), Technology in working order. Studies of work, interaction, and technology. London: Routledge, 35-54.

Heath, Christian/Paul Luff (1996), Convergent activities: Line control and passenger information on the London Underground, in: Engeström, Yrjö/David Middleton (Hrsg.), Cognition and communication at work. Cambridge: Cambridge University Press, 96-129.

Heath, Christian/Paul Luff (2000), Animating texts. The collaborative production of news stories, in: dies., Technology in action. Cambridge: Cambridge University Press, 61-86.

Heritage, John (1984), Garfinkel and ethnomethodology. Cambridge: Polity Press.

Heritage, John (1985), Analyzing news interviews. Aspects of the production of talk for an overhearing audience, in: van Dijk, Teun A. (Hrsg.), Handbook of discourse analysis. Vol. 3: Discourse and dialogue. London: Academic Press, 95-117.

Heritage, John (1998), Oh-prefaced responses to inquiry, in: Language in Society, Vol. 27, 291-334.

Heritage, John/Andrew L. Roth (1995), Grammar and institution. Questions and questioning in the broadcast news interview, in: Research on Language and Social Interaction, Vol. 28, Nr. 1, $1-60$.

Heritage, John/David Greatbatch (1986), Generating applause: A study of rhetoric and response at party political conferences, in: American Journal of Sociology, Vol. 92, 110-157. 
Heritage, John/David Greatbatch (1991), On the institutional character of institutional talk. The case of news interviews, in: Boden, Deirdre/Don H. Zimmerman (Hrsg.), Talk and social structure. Cambridge: Polity Press, 93-137.

Heritage, John/Steven Clayman/Don H. Zimmerman (1988), Discourse and message analysis. The micro-structure of mass media messages, in: Hawkins, Robert P./John M. Wiemann/Suzanne Pingree (Hrsg.), Advancing communication science: Merging mass and interpersonal processes. Sage Annual Review of Communication Research, Vol. 16, 77-109.

Hester, Stephen/Richard Fitzgerald (1999), Category, predicate and contrast: Some organizational features in a radio talk show, in: Jalbert, Paul L. (Hrsg.), Media Studies: Ethnomethodological approaches. Boston: University Press of America, 171-193.

Holly, Werner/Ulrich Püschel/Jörg Bergmann (Hrsg.) (2001), Der sprechende Zuschauer. Wie wir uns Fernsehen kommunikativ aneignen. Opladen: Westdeutscher Verlag.

Hutchby, Ian (1991), The organization of talk on talk radio, in: Scannell, Paddy (Hrsg.), Broadcast talk. London: Sage, 119-137.

Hutchby, Ian (1996a), Confrontation talk. Arguments, asymmetries and power on talk radio. Mahwah, NJ/London: Lawrence Erlebaum.

Hutchby, Ian (1996b), Power in discourse. The case of arguments on a British talk radio show, in: Discourse and Society, Vol. 7, Nr. 4, 481-497.

Hutchby, Ian (1997), Building alignments in public debates. A case study from British TV, in: Text, Vol. 17, Nr. 2 (Special Issue: Broadcast Talk, hrsg. von Joanna Thornborrow), 161-179.

Hutchby, Ian (1999), Rhetorical strategies in audience participation debates on radio and TV, in: Research on Language and Social Interaction, Vol. 32, Nr. 3, 243-267.

Hutchby, Ian (2001), „Witnessing“: The use of first-hand knowledge in legitimating lay opinions on talk radio, in: Discourse Studies, Vol. 3, Nr. 1, 481-497.

Jalbert, Paul L. (Hrsg.) (1999), Media Studies: Ethnomethodological approaches. Boston: University Press of America.

Jayyusi, Lena (1984), Categorization and the moral order. Boston, MA: Routledge and Keagan Paul.

Jayyusi, Lena (1988), Toward a socio-logic of the film text, in: Semiotica, Vol. 68, Nr. 3/4, 271-296.

Jefferson, Gail (1987), On exposed and embedded correction in conversation, in: Button, Graham/John R.E. Lee (Hrsg.), Talk and social organization. Clevedon: Multilingual Matters, 86-100.

Jensen, Klaus Bruhn (2002) (Hrsg.), A handbook of media and communication research. Qualitative and quantitative methodologies. London/New York: Routledge.

Jensen, Klaus Bruhn/Nicholas W. Jankowski (1991), A handbook of qualitative methodologies for mass communication research. London/New York: Routledge.

Keppler, Angela (1994), Der Gesprächsstoff der Medien, in: dies., Tischgespräche. Über Formen kommunikativer Vergemeinschaftung am Beispiel der Konversation in Familien. Frankfurt: Suhrkamp, 211-267.

Klemm, Michael (2000), Zuschauerkommunikation: Formen und Funktionen der alltäglichen kommunikativen Fernsehaneignung. Frankfurt am Main: Lang.

Knoblauch, Hubert/Jürgen Raab (2002), Der Werbespot als kommunikative Gattung, in: Willems, Herbert (Hrsg.), Die Gesellschaft der Werbung. Kontexte und Texte. Produktionen und Rezeptionen. Entwicklungen und Perspektiven. Opladen: Westdeutscher Verlag, 139-154.

Lee, John (1984), Innocent victims and evil-doers, in: Women's Studies International Forum, Vol. 7, Nr. 1, 69-73.

Liddicoat, Anthony/Annie Brown/Susanne Döpke/Kristina Love (1992), The effect of the institution: Openings in talkback radio, in: Text, Vol. 12, Nr. 4, 541-562.

Luckmann, Thomas (1986), Grundformen der gesellschaftlichen Vermittlung des Wissens: Kommunikative Gattungen, in: Neidhardt, Friedhelm/M. Rainer Lepsius/Johannes Weiß (Hrsg.), Kultur und Gesellschaft. Kölner Zeitschrift für Soziologie und Sozialpsychologie, Sonderheft 27. Opladen: Westdeutscher Verlag, 191-211.

Luff, Paul/Jon Hindmarsh/Christian Heath (2000) (Hrsg.), Workplace studies: Recovering work, practice and informing system design. Cambridge: Cambridge University Press. 
Lynch, Michael (2000a), The ethnomethodological foundations of conversation analysis, in: Text, Vol. 29, Nr. 4, 517-532.

Lynch, Michael (2000b), Response to Wes Sharrock, in: Text, Vol. 29, Nr. 4, 541-544.

Matthewson, Lisa (1992), Talking to the TV. The conversational behavior of television viewers, in: Wellington Working Papers in Linguistics, Nr. 5, 17-35.

McHoul, Alec W. (1987), An initial investigation of the usability of fictional conversation for doing conversation analysis, in: Semiotica, Vol. 67, Nr. 1/2, 83-104.

Meier, Christoph (1997), Arbeitsbesprechungen. Interaktionsstruktur, Interaktionsdynamik und Konsequenzen einer sozialen Form. Opladen: Westdeutscher Verlag.

Meier, Christoph (2000a), Neue Medien - neue Kommunikationsformen? Strukturmerkmale von Videokonferenzen, in: Kallmeyer, Werner (Hrsg.), Sprache und neue Medien. Institut für Deutsche Sprache, Jahrbuch 1999. Berlin: de Gruyter, 195-217.

Meier, Christoph (2000b), Videokonferenzen - Beobachtungen zu Struktur und Dynamik und Folgen einer neuen Kommunikationsform, in: Boos, Margarete/Kai J. Jonas/Kai Sassenberg (Hrsg.), Virtuelle Kommunikation in Organisationen. Theorie - psychologische Technologie - Praxiserfahrungen. Göttingen: Hogrefe, 153-163.

Meier, Christoph (2002), Kommunikation in räumlich verteilten Teams: Videokonferenzen bei Technics, in: Thimm, Caja (Hrsg.), Unternehmenskommunikation offline/online. Wandelprozesse interner und externer Kommunikation durch neue Medien. Frankfurt u.a.: Lang, 103-134.

Meier, Christoph (2003), Doing "groupness" in a spatially distributed workgroup: The case of videoconferences at Technics, in: Frey, Lawrence R. (Hrsg.), Group communication in context. Studies of bona fide groups (second edition). Mahwah, NJ/London: Lawrence Erlbaum, 367-397.

Miller, Toby/Alec McHoul (1998), Popular culture and everyday life. London: Sage.

Montgomery, Martin (1986), DJ talk, in: Media, Culture \& Society, Vol. 8, 421-440.

Montgomery, Martin (1991), „Our Tune“. A study of a discourse genre, in: Scannell, Paddy (Hrsg.), Broadcast talk. London: Sage, 138-177.

Myers, Greg (2000), Entitlement and sincerity in broadcast interviews about Princess Diana, in: Media, Culture \& Society, Vol. 22, 167-185.

Nofsinger, Robert E. (1988/1989), „Let’s talk about the record“: Contending over topic redirection in the Rather/Bush interview, in: Research on Language and Social Interaction, Vol. 22, 273-292.

Pomerantz, Anita (1984), Agreeing and disagreeing with assessments: some features of preferred/ dispreferred turn shapes, in: Atkinson, J. Maxwell/John C. Heritage (Hrsg.), Structures of social action. Studies in conversation analysis. Cambridge: Cambridge University Press, $57-101$.

Pomerantz, Anita (1988/1989), Constructing skepticism. Four devices used to engender the audience's skepticism, in: Research on Language and Social Interaction, Vol. 22, $293-314$.

Rama Martinez, Maria Esperanza (1993), Preference organization in broadcast political interviews, in: Verba, Vol. 20, 45-60.

Roth, Andrew L. (1998), Who makes the news? Descriptions of television news interviewees' public personae, in: Media, Culture \& Society, Vol. 20, Nr. 1, 79-107.

Roth, Andrew L. (2002), Social epistemology in broadcast news interviews, in: Language in Society, Vol. 31, 355-381.

Sacks, Harvey (1972), An initial investigation of the usability of conversational materials for doing sociology, in: Sudnow, David (Hrsg.), Studies in social interaction. New York: The Free Press, $31-74$.

Sacks, Harvey (1984a), Notes on methodology, in: Atkinson, J. Maxwell/John C. Heritage (Hrsg.), Structures of social action. Studies in conversation analysis. Cambridge: Cambridge University Press, 21-27.

Sacks, Harvey (1984b), On doing „being ordinary“, in: Atkinson, J. Maxwell/John C. Heritage (Hrsg.), Structures of social action. Studies in conversation analysis. Cambridge: Cambridge University Press, 413-429. 
Sacks, Harvey (1992), Lectures on conversation. Band 1 und 2. Hrsg. von Gail Jefferson. Oxford: Blackwell.

Sacks, Harvey/Emanuel A. Schegloff/Gail Jefferson (1974), A simplest systematics for the organization of turn-taking for conversation, in: Language, Vol. 50, 696-735.

Scannell, Paddy (1997), Saying and showing. A pragmatic and phenomenological study of a television documentary, in: Text, Vol. 17, Nr. 2 (Special Issue: Broadcast Talk, hrsg. von Joanna Thornborrow), 225-240.

Scannell, Paddy (Hrsg.) (1986), Broadcast talk (Media, Culture \& Society, special issue), Vol. 8, Nr. 4.

Scannell, Paddy (Hrsg.) (1991), Broadcast talk. London: Sage.

Schegloff, Emanuel A. (1972), Sequencing in conversational openings, in: Gumperz, John J./Dell Hymes (Hrsg.), Directions in sociolinguistics. The ethnography of communication. New York: Holt, Rinehart \& Winston, 346-380 (orig. in: American Anthroplogist, 1968, Vol. 70, Nr. 6, 1075-1095).

Schegloff, Emanuel A. (1982), Discourse as an interactional achievement: Some uses of „uh huh“ and other things that come between sentences, in: Tannen, Deborah (Hrsg.), Analyzing disourse: Text and talk. Georgetown University round table on languages and linguistics 1981.

Schegloff, Emanuel A. (1988/1989), From interview to confrontation: Observations on the Bush/Rather encounter, in: Research on Language and Social Interaction, Vol. 22, 215-240.

Schegloff, Emanuel A. (1992), Introduction, in: Sacks, Harvey (1992), Lectures on Conversation. Band 1. Hrsg. von Gail Jefferson. Oxford: Blackwell, ix-lxii.

Schegloff, Emanuel A. (1999a), „Schegloff's texts“ as „Billig’s data“: A critical reply to Billig, in: Discourse and Society, Vol. 10, 558-571.

Schegloff, Emanuel A. (1999b), Naivete vs. sophistication or discipline vs. self-indulgence: A rejoinder to Billig, in: Discourse and Society, Vol. 10, 577-582.

Schegloff, Emanuel A. (2000), Overlapping talk and the organization of turn-taking for conversation, in: Language in Society, Vol. 29, Nr. 1, 1-64.

Schegloff, Emanuel A./Harvey Sacks (1973), Opening up closings, in: Semiotica, Vol. 8, 289-327.

Schegloff, Emanuel A./Irene Koskik/Sally Jacoby/David Olsher (2002), Conversation analysis and applied linguistics, in: Annual Review of Applied Linguistics, Vol. 22, 3-31.

Schenkein, Jim (1979), The radio raiders story, in: Psathas, George (Hrsg.), Everyday language: Studies in ethnomethodology. New York: Irvington, 187-201.

Schmidt, Gurly (2000), Chat-Kommunikation im Internet - eine kommunikative Gattung? in: Thimm, Caja (Hrsg.), Soziales im Netz: Sprache, Beziehungen und Kommunikationskulturen im Internet. Opladen: Westdeutscher Verlag, 109-130

Selting, Margret/Elizabeth Couper-Kuhlen (2001), Forschungsprogramm „Interaktionale Linguistik“, in: Linguistische Berichte, Vol. 187, 257-287.

Sharrock, Wes (2000), Where the simplest systematics fits: A response to Michael Lynchs's „The ethnomethodological foundations of conversation analysis“, in: Text, Vol. 29, Nr. 4, 533-544.

Stetson, Jeff (1999), Victim, offender and witness in the emplotment of news stories, in: Jalbert, Paul L. (Hrsg.), Media Studies: Ethnomethodological approaches. Boston: University Press of America, 77-110.

Suchman, Lucy (1987), Plans and situated actions: The problem of human-machine communication. Cambridge: Cambridge University Press.

Suchman, Lucy (1993), Technologies of accountability. Of lizards and aeroplanes, in: Button, Graham (Hrsg.), Technology in working order. Studies of work, interaction, and technology. London: Routledge, 113-126.

ten Have, Paul (1999), Doing conversation analysis: A practical guide. London: Sage.

Thimm, Caja (Hrsg.) (2000), Soziales im Netz: Sprache, Beziehungen und Kommunikationskulturen im Internet. Opladen: Westdeutscher Verlag.

Thornborrow, Joanna (1997), Having their say. The function of stories in talk-show discourse, in: Text, Vol. 17, Nr. 2 (Special Issue: Broadcast Talk, hrsg. von Joanna Thornborrow), 241-262.

Tuchman, Gaye (1972), Objectivity as strategic ritual, in: American Journal of Sociology, Vol. 77, 660-679. 
Tuchman, Gaye (1973), The technology of objectivity: Doing „objective“ TV news film, in: Urban Life and Culture, Vol. 2, 3-26.

Ulmer, Bernd/Jörg R. Bergmann (1993), Medienrekonstruktionen als kommunikative Gattungen? in: Holly, Werner/Ulrich Püschel (Hrsg.), Medienrezeption als Aneignung. Methoden und Perspektiven qualitativer Medienforschung. Opladen: Westdeutscher Verlag, 81-102.

Whalen, Jack/Marilyn Whalen/Kathryn Henderson (2002), Improvisional choreography in teleservice work, in: British Journal of Sociology, Vol. 53, Nr. 2, 239-258. 